\title{
Clinical profile, viral load, management and outcome of neonates born to COVID 19 positive mothers: a tertiary care centre experience from India
}

\author{
Pratima Anand $^{1} \cdot$ Anita Yadav $^{1} \cdot$ Pradeep Debata $^{1}$ (D) $\cdot$ Sumitra Bachani ${ }^{2} \cdot$ Nitesh Gupta $^{3} \cdot$ Rani Gera $^{1}$
}

Received: 17 July 2020 / Revised: 27 August 2020 / Accepted: 31 August 2020 / Published online: 10 September 2020

(C) Springer-Verlag GmbH Germany, part of Springer Nature 2020

\begin{abstract}
Despite rapidly evolving knowledge about COVID 19 infection, routes of perinatal COVID 19 transmission and viral load in mother neonate dyad remain uncertain. Data were analysed to describe the clinicodemographic profile and viral load in neonates born to COVID 19 positive mothers. Of 2947 deliveries, 69 mothers were COVID 19 positive (2.3\%), with 1 abortion, 2 macerated stillbirths and 2 fresh stillbirths as pregnancy outcomes. Of 65 tested neonates, 10.7\% (7) were confirmed COVID 19 positive by RTPCR (reverse transcriptase-polymerase chain reaction). Viral load (cycle threshold, Ct of E, RDRp) in neonates was comparable with the Ct reported from adults; however, neonates had milder clinical manifestations. All 7 neonates who tested positive for COVID 19 were subsequently discharged. Six of the 7 neonates were asymptomatic and 1 neonate needed respiratory support (indication being prematurity) which resolved after $48 \mathrm{~h}$. Maternal and neonatal comparison of $\mathrm{Ct}$ of $\mathrm{E}$ and RdRp gene was statistically non-significant $(25.97$ vs $19.68, p=0.34$ and 26.5 vs $25.0, p=0.84)$. Viral loads of mothers with COVID 19 positive neonates compared with mothers with COVID 19 negative neonates for $\mathrm{E}$ and RdRp gene were also statistically non-significant ( 25 vs $27.19, p=0.63$ and 19.6 vs $27.6, p=0.08$ ). The majority (93\%) of neonates tested later than $48 \mathrm{~h}$ (roomed in with mother and breastfed) tested negative.

Conclusion: The study supports milder manifestation in COVID 19 positive neonates. Risk of transmission from COVID 19 positive mother to neonate by rooming-in and breastfeeding is low. In this study on a limited number of neonates, maternal viral load was not found to be associated with the positivity status or severity of the illness of neonate.
\end{abstract}

Communicated by Daniele De Luca

Electronic supplementary material The online version of this article (https://doi.org/10.1007/s00431-020-03800-7) contains supplementary material, which is available to authorized users.

Pradeep Debata

drpkdebata@gmail.com

Pratima Anand

anandpratima@gmail.com

Anita Yadav

ann_y26@yahoo.com

Sumitra Bachani

sumitrabachani@gmail.com

Nitesh Gupta

niteshgupta2107@gmail.com
Rani Gera

drranigera@gmail.com

1 Department of Paediatrics, Vardhman Mahavir Medical College \& Safdarjung Hospital, New Delhi 110023, India

2 Department of Obstetrics and Gynaecology, Vardhman Mahavir Medical College \& Safdarjung Hospital, New Delhi, India

3 Department of Respiratory Medicine, Vardhman Mahavir Medical College \& Safdarjung Hospital, New Delhi, India 
What is Known:

- Neonates born to COVID 19 positive mothers are at risk of COVID 19 infection.

What is New:

- Risk of transmission of COVID 19 from mother to neonate, with rooming-in and breastfeeding, appears low.

- In this study on a limited number of neonates, maternal viral load of COVID 19 (E and RdRp cycle thresholds) was not associated with severity of illness or COVID 19 positivity in neonates.

Keywords Neonates $\cdot$ COVID $19 \cdot$ Viral load $\cdot$ Clinical profile

$\begin{array}{ll}\begin{array}{l}\text { Abbreviations } \\ \text { CDC }\end{array} & \begin{array}{l}\text { Centre for Disease Control and Prevention } \\ \text { COVID 19 }\end{array} \\ \text { Ct } & \begin{array}{l}\text { Cycle threshold } \\ \text { Envelope gene }\end{array} \\ \text { E gene } & \text { Food and Drug Administration } \\ \text { FDA } & \text { High dependency unit } \\ \text { HDU } & \begin{array}{l}\text { Open reading frame 1b non-structural } \\ \text { protein } 14\end{array} \\ \text { RdRp gene } & \text { RNA-dependent RNA polymerase gene } \\ \text { RTPCR } & \text { Reverse transcriptase-polymerase } \\ & \text { chain reaction } \\ \text { SARS CoV } & \text { Severe acute respiratory syndrome } \\ & \begin{array}{l}\text { coronavirus 2 } \\ \text { Small for gestation }\end{array}\end{array}$

\section{Background}

Towards the end of 2019, a novel coronavirus was identified to cause a cluster of cases of pneumonia in China. With the rapid spread, the number of cases has increased globally. In February 2020, the World Health Organisation (WHO) designated the disease COVID 19, which stands for coronavirus disease 2019. To date, India has reported 366,946 confirmed cases of COVID 19 infection [1]. Pregnant women are a vulnerable group of the population susceptible to the COVID 19 infection due to physiological changes in the immunological and circulatory parameters. As a result, neonates born to COVID 19 positive mothers are at risk of infection as well as adverse outcomes due to the infection. Little is known about perinatal and neonatal COVID 19 infection until now, with current evidence-based predominantly on case series and case reports. One of the first and largest case series is on 33 neonates born to COVID 19 confirmed mothers [2]. Multiple routes of transmission apart from post-partum transmission (horizontal) have been postulated including trans placental in utero, the immediate peripartum period through fetoplacental bleed or amniotic fluid and breast milk [3-28].

The first study describing the clinical characteristics and investigating the possibility of vertical transmission of SARS-CoV-2 in nine pregnant women with laboratory- confirmed COVID 19, demonstrated lesser severity and no evidence of vertical transmission [22, 23]. However, recently, severe illness in the antenatal period and pregnant women has been reported in the literature. Ellington et al described that amongst women with COVID 19, approximately one-third of pregnant women were hospitalised compared with $5.8 \%$ of non-pregnant women [13]. Similarly, higher risk of decompensation and complications were reported in the latter half of pregnancy by Badr et al. [14] Most recently, reports with evidence of vertical transmission have been published [28, 29]. Vivanti et al demonstrated the transplacental transmission, confirmed by comprehensive virological tests in the placenta, along with symptoms and clinical manifestations in the neonate [29].

With a rising number of cases and a shortage of hospital beds, the concept of cycle threshold $(\mathrm{Ct})$ indicating the viral load has been studied. Progressive decrease of viral load over time is observed in all studies conducted in COVID 19 patients with positive detection being observed until 17-21 days after onset of symptoms, independently of symptoms [30]. Timing of testing and sensitivity of reverse transcriptase-polymerase chain reaction (RTPCR) on nasopharyngeal samples continues to remain a topic of debate.

The study was conducted to describe the clinical profile of neonates born to mothers who tested positive for COVID 19 infection and to determine the association of neonatal COVID 19 status and viral load with maternal clinical status and viral load.

\section{Material and methods}

\section{Study setting and design}

Neonates born to COVID 19 positive mothers from 1st April 2020 to 10th July 2020 were included in analysis. The hospital is a teaching and academic medical college and tertiary referral centre for neonatal care. The annual delivery rate is approximately 25,000 , with nearly 4800 NICU admissions per year. The resuscitation area and 
team composition were as per current guidelines with all precautions against COVID 19 infection. Delayed cord clamping and immediate skin to skincare were practised as per national and ILCOR neonatal resuscitation guidelines in all vigorous neonates. The gestational assessment was done by the best estimate of the last menstrual period (LMP), first-trimester ultrasound or by Ballard examination (in case LMP and ultrasound both were unavailable). Intrauterine growth status at birth as appropriate for gestation (AGA), small for gestation (SGA) and large for gestation (LGA) was assessed by using $\mathrm{z}$ scores on Intergrowth 21 st neonatal anthropometric charts. Neonates with a birth weight below 2SD (standard deviation) $\mathrm{z}$ scores were classified as small for gestation.

COVID 19 positive and COVID 19 negative mother neonate dyads were provided care in two different locations, situated in two different buildings of the hospital, respectively. COVID block with a dedicated labour room and maternity OT was earmarked for delivery of COVID 19 positive women presenting to the hospital for delivery. Within the COVID block, an isolation area was kept for delivery of women who were suspected to have COVID 19 infection (presenting from containment area or symptomatic) but were awaiting the report. The COVID block of the hospital was not equipped with negative pressure, however air conditioning with 12 air exchanges per hour, as per recommendations was followed (online supplement 1 and 2).

All the other women who were either not suspected to have COVID 19 infection or were COVID 19 negative on testing, were delivered in the non-COVID block. Any mother/neonate who developed symptoms after the delivery was immediately shifted to the COVID block. The sample for RTPCR was also taken at the same time.

So, there were five separate designated areas for the care of mother neonate dyad with suspected or confirmed COVID 19 infection: (i) non-COVID NICU in the non-COVID building, (ii) non-COVID postnatal wards where mother and neonate without COVID 19 infection were roomed in, (iii) COVID neonatal unit for neonates needing NICU care (born to COVID 19 positive mothers and awaiting COVID 19 report), (iv) COVID block postnatal wards where stable neonates (born to COVID 19 positive mothers and awaiting COVID 19 reports) were roomed in with mother, (v) COVID 19 positive ICU and ward where neonates positive for COVID 19 were cared for, either with mother, if stable or in COVID NICU if they required NICU care.

As per the recommendations, all the health care staff followed donning and doffing sequence of complete personal protective equipment (PPE) which included goggles, cap, double layer gloves, N95 mask, shoe covers and overall. Face shields were also used in areas where possible aerosol-generating procedures were done (online supplement 3).

\section{Clinical care and discharge policy}

All stable neonates born to COVID 19 confirmed mothers were roomed in and breastfed ensuring all universal and contact precautions against COVID 19 infection. If the neonate tested negative, the mother neonate dyad was discharged once discharge criteria were met.

Neonates born to COVID 19 positive mothers and requiring NICU care for any reason (comorbidity like prematurity, low birth weight, or transient tachypnoea of neonate) were nursed in separate designated NICU in COVID block. COVID 19 positive neonates, if requiring NICU care, were managed in another separate COVID ICU; stable COVID 19 positive neonates were roomed in with mother and breastfed.

The protocol for clinical management and investigation of neonates was based on national guidelines and recommendations as per the currently available evidence [31-34]. Neonates who tested positive for COVID 19 infection were not given any specific treatment against COVID 19 infection. The standard operating procedure (SOP) for the management of neonates born to mothers with suspected or confirmed COVID 19 infection is described in the online supplement 4.

Discharge of the mother neonate dyad was done as per existing national operational guidelines for COVID 19; stable neonates were kept for a minimum of 48 to $72 \mathrm{~h}$ and discharged once the mother was afebrile for 3 days. All mothers and caregivers were counselled in detail at discharge regarding precautions and self-protection against COVID 19 infection at home. Telephonic follow-up at 14th and 29th day was done for all positive neonates.

\section{Testing strategy}

As per national guidelines by expert group consensus and unit policy, all neonates born to COVID 19 positive mothers were tested [31-34].

As per the testing guidelines issued by the national expert groups, antenatal testing was done if the mother resided in any of the containment zones or if the mother had any of the symptoms suggestive of COVID 19 infection. Universal screening of antenatal mothers was not done as it is not recommended as per national policy on COVID 19, until writing of this paper.

Neonates born to mothers who were diagnosed before delivery were tested within $24 \mathrm{~h}$ of birth. Neonates born to mothers detected COVID 19 positive after delivery, during the hospital stay, underwent testing as soon as mother was confirmed positive. Samples (nasopharyngeal/oropharyngeal swabs) were collected under aseptic precautions, after cleaning the neonate. No other sample (blood, anal or rectal swabs) was collected from the neonate. CSF examination to exclude CNS invasion in neonates who tested positive for COVID 19, was also not a part of management protocol in 
the unit. Nasopharyngeal samples were taken under strict aseptic precautions, by the paediatric resident, with a postgraduate qualification. They all underwent training on the technique of appropriate sampling method and collection.

Also, autopsy and fetal tissue examination of stillbirths and abortus was not a part of the unit protocol for the investigation of COVID 19 infection.

Reverse transcriptase-polymerase chain reaction (RTPCR) was the method used to diagnose COVID 19 infection in mother and neonate. Indian Council of Medical Research (ICMR) approved kit (Lab Gun kit) was used for the testing. The kit, manufactured by Labgenomics (Korea), is approved by Food and Drug Administration under Emergency Use Authorization (EUA), and hence conforms to the CDC recommendations [35]. Primer probes were run through initial screening for E (envelope) gene-specific to Sarbeco subgenus. Samples positive in the first screening were subjected to confirmatory test targeting two genes, one SARS CoV 2 specific RdRp (RNA-dependent RNA polymerase) gene and other Sarbeco subgenus ORF-1b-nsp14b gene. A positive test was labelled when samples positive for either of the above two genes. The detection limit was 35 for E, RDRp and ORF genes [36]. The mean turnaround time for PCR was $24 \mathrm{~h}( \pm 8 \mathrm{~h})$.

\section{Enrolment and data collection}

The medical records of all mothers who delivered in the COVID facility from 1st April 2020 to 10th July 2020 were reviewed independently. All neonates born to COVID confirmed mothers were enrolled. Data were extracted from the patient records and included address, age, gestational age, comorbidities, symptoms at onset, the outcome of pregnancy and information on neonates (including birth weight, Apgar score, perinatal complications and clinical course during the hospital stay.

Also noted were the time from onset of symptoms to diagnosis, vital signs on admission, laboratory tests (Ct values), treatments received (antiviral therapies, antibiotics, corticosteroids, anticoagulants) and duration of hospital stay. Maternal and neonatal deaths amongst this cohort were also audited.

\section{Statistical analysis}

The SPSS program performed the statistical analysis for Windows, version 21. Continuous variables are presented as mean $\pm \mathrm{SD}$, and categorical variables are presented as absolute numbers and percentages. Data were checked for normality before statistical analysis. Normally distributed continuous variables were compared using the unpaired t-test, whereas the Mann-Whitney $U$ test was used for those variables that were not normally distributed. For all statistical tests, a $p$ value of less than 0.05 was taken to indicate a significant difference.

\section{Ethics approval}

The study was approved by the Institutional Ethics Committee vide letter number IEC/VMMC/SJH/Project/2020-07/CC-21.

\section{Results}

Of the total 2947 deliveries during the study period, 69 mothers were confirmed COVID 19 positive (2.3\%). There were 1 abortion, 2 macerated stillbirths and 2 fresh stillbirths as pregnancy outcomes. The stillbirth rate of the cohort was 57 per 1000 total births (4/69). Elective caesarean delivery was performed in 09(13.4\%) while $17(25.3 \%)$ underwent emergency caesarean delivery for various fetomaternal indications, commonest being fetal distress. COVID 19 positive status did not influence the mode of termination.

A total of $18(26 \%)$ mothers were asymptomatic and tested for COVID 19 as they presented from containment area (which has a higher risk of COVID 19 transmission due to a cluster of COVID 19 positive cases) of New Delhi. A total of 51 of the 69 (74\%) mothers had symptoms and majority (45/ 69 ; 65\%) of mothers had mild symptoms of COVID 19 infection with low-grade fever, cough and/or diarrhoea. A total of 39 of the 69 positive mothers (56.5\%) were detected positive in the postpartum period (Fig. 1).

Of the 65 neonates (63 singletons and 1 twin pregnancy live births) born to COVID 19 positive mothers, 7 tested positive for COVID 19. Viral loads (Ct of E and RdRp genes) were available in 50 of the 69 positive mothers and 5 of the 7 positive neonates.

The demographic details of the cohort are described in Table 1. Approximately one-third cohort was born preterm $(26 / 65 ; 40 \%)$. The mean maternal age was $26.7 \pm 4.54$ years. $50.9 \%$ were primigravida. Mean gestation $( \pm \mathrm{SD})$ and mean neonatal birth weight $( \pm \mathrm{SD})$ were $36.47 \pm 2.65$ weeks and $2523 \pm 722.12$ g respectively. $55.3 \%$ of neonates were females.

\section{Individual details}

Neonate 1 and neonate 4 were confirmed COVID 19 positive within $24 \mathrm{~h}$ of birth. Neonate 2 and 3 were born preterm and low birth weight. Six of the 7 neonates were asymptomatic and one neonate (neonate 5) received respiratory support (CPAP) for $48 \mathrm{~h}$. The indication for respiratory support was prematurity (Fig. 1). All 7 neonates remained haemodynamically stable during the hospital stay and were subsequently discharged (Table 2). 


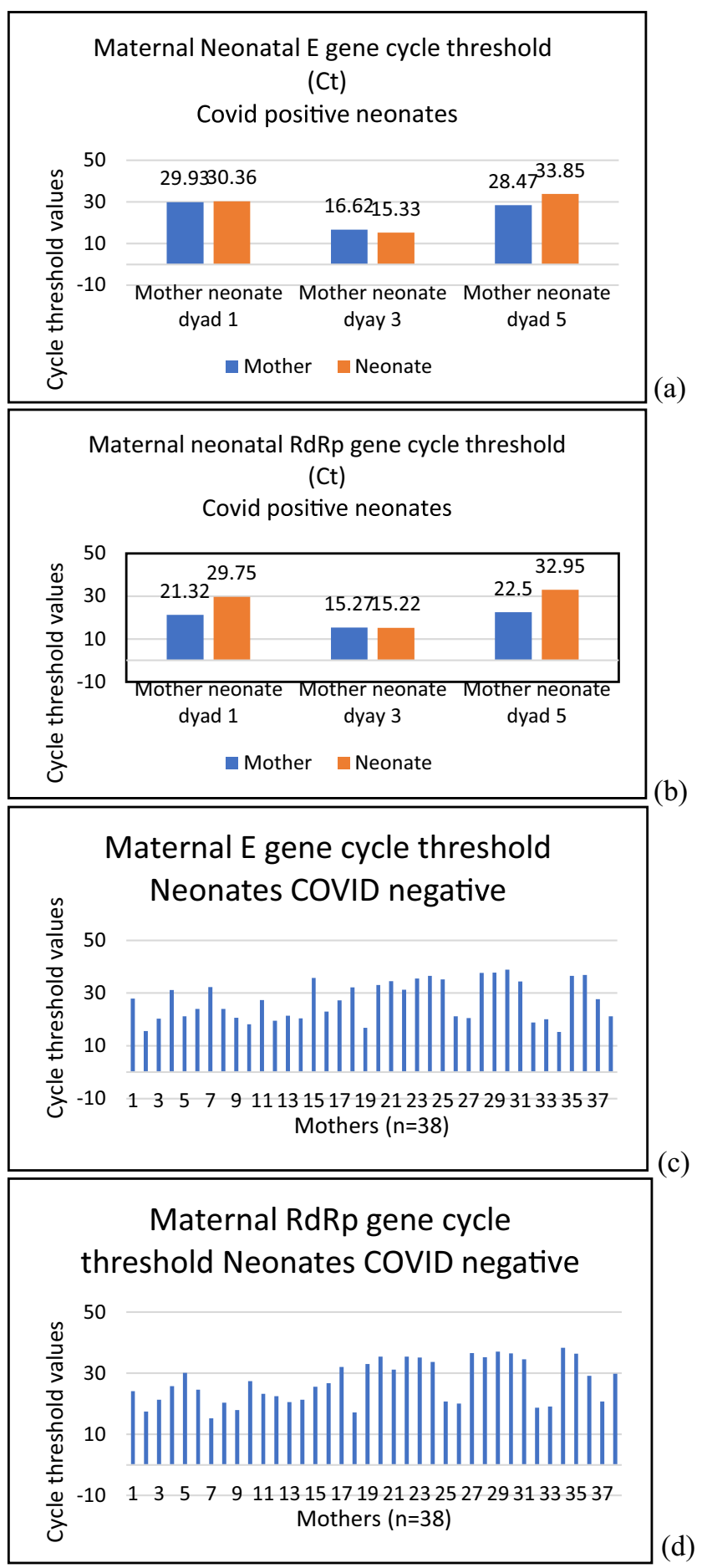

Fig. 1 Maternal and neonatal viral load of COVID 19 (cycle thresholds of E, RdRp and ORF-1b-nsp14 based assay). a E gene cycle thresholds in maternal neonatal dyads in COVID positive neonates. $\mathbf{b} \mathrm{RdRp}$ gene cycle thresholds in maternal neonatal dyads in COVID positive neonates. $\mathbf{c} \mathrm{E}$ gene cycle thresholds in COVID mothers whose neonates were negative. d RdRp gene cycle thresholds in COVID positive mothers whose neonates were negative

The mother of neonate 1 was critical immediately after delivery, received intensive care and died subsequently on day 3 post-partum. Neonate was nursed in COVID ICU
Table 1 Demographic characteristics of cohort $\left(N=65^{*}\right)$

\begin{tabular}{lll}
\hline S. No & Variable & $N(\%)$ \\
\hline 1 & Number of neonates tested positive & $7(13.8 \%)$ \\
& Symptomatic & 2 \\
& Asymptomatic & 5 \\
2 & Mode of delivery (LSCS) & $26(40.0 \%)$ \\
3 & Female gender & $36(55.3 \%)$ \\
4 & Multiple gestation & $1(1.5 \%)$ \\
5 & Preterm (<37 weeks) & $26(40.0 \%)$ \\
6 & Low birth weight (<2500 g) & $22(33.8 \%)$ \\
7 & Mean maternal age (years) (SD) & $26.7 \pm 4.54$ \\
8 & Primigravida & $29(44 \%)$ \\
9 & Mean gestation (weeks) & $36.47 \pm 2.65$ \\
10 & Mean birth weight (g) & $2523 \pm 722$ \\
11 & Deaths (total) & $2(\%)$ \\
& COVID positive & 0 \\
& COVID negative & 2 \\
\hline
\end{tabular}

*Does not include 1 abortion and 4 stillbirths

(intensive care unit) since birth and subsequently discharged. The cause of maternal death was a pulmonary embolism (a complication of COVID 19).

The mother of neonate 2 was tested because of fever 13 days before delivery and was confirmed COVID 19 positive twice, the second test done 4 days before delivery. However, the third test one day before delivery was negative. Neonate was delivered by caesarean delivery because of fetal distress, had to be separated from mother at birth because of prematurity and received care in NICU. Since mother died on day 11 postpartum and strong suspicion of COVID related cause was kept as a possibility by obstetricians, the neonate was tested before discharge on day 14 of life. The cause of maternal death was severe metabolic acidosis due to acute on chronic renal failure (COVID 19 related death).

The mother of neonate 3 developed symptoms on day 4 postpartum, following which she was tested and confirmed positive. Neonate was nursed with mother immediately after birth and was positive for COVID 19 when tested on day 4 of life.

The mother of neonate 4 was tested before delivery due to the presence of fever. Mother was reported to be COVID 19 positive before delivery and hence neonate tested within $24 \mathrm{~h}$ of birth. Until the report of the neonate was awaited, the mother neonate dyad was roomed in and breastfeeding was initiated and continued.

The mother of neonate 5 tested positive for COVID 19 on day 4 postpartum. Neonate had respiratory distress at birth and hence had to be separated from mother, was tested on day 4 once the mother was confirmed COVID 19 positive. The neonate was being nursed in NICU when the mother developed symptoms. 


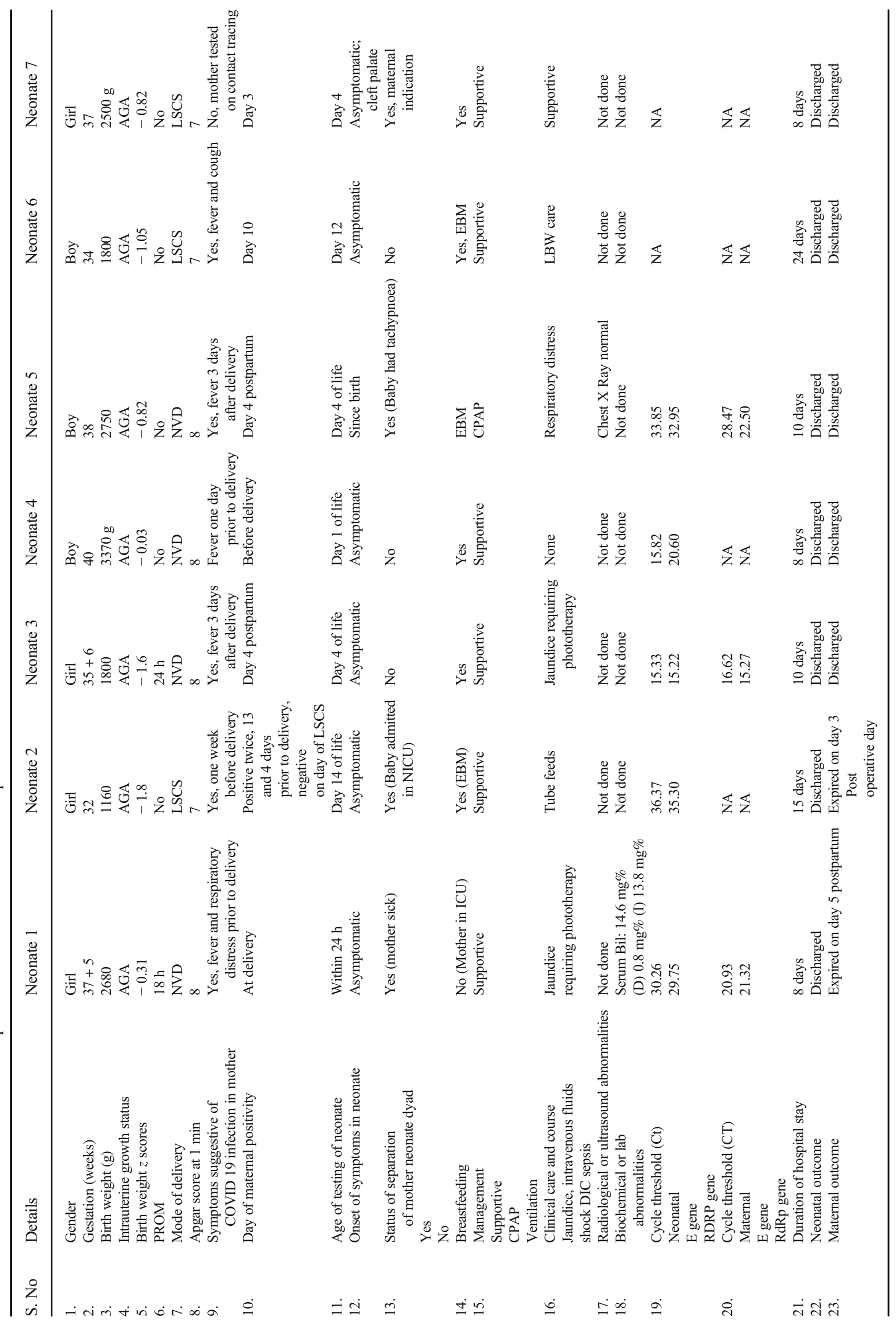


The mother of neonate 6 developed symptoms on day 12 postpartum and tested positive for COVID 19 infection. Neonate had to be separated at birth due to maternal and neonatal indication, however, when tested subsequently due to maternal positivity, neonate also tested positive on day 14 of life.

The mother of neonate 7 was tested on day 4 postpartum due to the presence of symptoms of COVID 19 infection. Neonate was nursed along with the mother and tested positive when tested on day 4.

All COVID 19 positive neonates remained asymptomatic as per the last follow-up telephonic call on day 14/day 29 of life.

Viral load as assessed by cycle threshold $(\mathrm{Ct})$ of the RTPCR for 5 neonates was highest for neonate 3 and neonate 4 (Ct 15.82 and 15.33) whereas neonate positive on day 14 had almost below detection level $\mathrm{Ct}$ (36.37). The mean value of neonatal E gene assay was $26.5 \pm 9.8 \mathrm{vs}$ mean in mothers of $25.0 \pm 7.2 ; p$ value of 0.84 . The mean value of neonates $\mathrm{RdRp}$ gene assay was $25.97 \pm 9.44$ vs mean in mothers of $19.69 \pm$ 3.8; $p$ value of 0.34 (Fig. 1).

The individual clinical and viral load details for the COVID 19 positive neonates are described in Table 2.

Neonatal deaths There were two deaths in the cohort; both neonates tested COVID 19 negative (online supplement 5). COVID 19 negative status was established on basis of single nasopharyngeal swab RTPCR. Mothers of both the above neonates were symptomatic (mild symptoms). Autopsy of the two neonates could not be done and placental and amniotic fluid examination for COVID 19 infection, at the time of birth could not be done.

All factors analysed in the comparative between COVID 19 positive and COVID 19 negative neonates were statistically non-significant. Mode of delivery (LSCS) was not statistically different ( $p$ value 0.25 ) in COVID 19 positive neonates vs COVID 19 negative neonates (Table 3).

Of the overall cohort, 25 (38\%) neonates were tested within $24 \mathrm{~h}$ of birth; rest 40 were tested beyond $48 \mathrm{~h}$ of life when the mother was confirmed COVID 19 positive. A total of $93 \%$ (35) of the 38 neonates tested beyond $48 \mathrm{~h}$, who were roomed in with mother and breastfed, were negative (Fig. 2). Fourteen mother neonate dyads from the overall cohort needed separation at birth as per indications. The 14 neonates needed NICU care for other non-COVID 19 neonatal comorbidities, which included prematurity, low birth weight care and jaundice. All the neonates remained haemodynamically stable and were subsequently discharged.

\section{Discussion}

The study describes 7 COVID 19 positive neonates of the total 65 neonates tested ( $10.7 \%$ positivity). None of the COVID 19 positive neonates developed severe manifestations of the disease and were discharged subsequently. The unit followed the policy of rooming in with mother and direct breastfeeding with precautions in stable neonates born to COVID 19 positive mothers. A total of $93 \%$ of the roomed neonates tested negative for COVID 19 and remained stable throughout the hospital stay. The positivity rate in neonates is variable in previous reports. In a multicentric study from Turkey [37], the rate has been described as $3.3 \%$, whereas in a systematic review [38] and 2 other studies [2, 39], it is observed to be $2.7 \%(1 / 37)$ to $9.99 \%(3.3 \%)$ and $4.2 \%(3 / 72)$.

Neonates are precarious as they are at risk of transmission of COVID 19 infection (i) in utero through the placenta, (ii) in the immediate peripartum period through amniotic fluid aspiration and fetoplacental bleed and lastly (iii) through breastfeeding. Hence once a neonate is born to COVID 19 positive mother, all possible routes of transmission should be taken into consideration.

Recent evidence suggests vertical transmission of COVID 19, as reported by Vivanti et al. [29] The case report described signs of acute and chronic intervillous inflammation consistent with the severe systemic maternal inflammatory status triggered by SARS CoV 2 and demonstration of virus in neonatal samples associated with neurological symptoms thus implying transplacental transmission of SARS CoV 2. Another case report has also raised the concern of positive amniotic fluid for COVID 19 and positive antibody titres in neonates immediately at birth [23]. Similar case of positive amniotic fluid followed by pneumonia in the neonate is also observed by Zeng et al. [26] Few earlier reports, however, failed to demonstrate the vertical transmission from mother to neonate by placental and fetal tissue examination [40-42].

In the current study, the possibility of vertical transmission can be debated because RTPCR was reported positive in 4 neonates who were either separated from mother at birth and nursed in NICU (neonate 1, 2 and 5) or were tested within $24 \mathrm{~h}$ of birth (neonate 4 ).

The case of neonate 2 is even more intriguing where the mother was reported positive twice before delivery, the last report being 4 days before delivery. Despite being negative on the day of delivery, and neonate being nursed in NICU separate from mother, neonate tested positive on day 14 of life. Since the mother tested negative just before delivery, as per the existing guidelines, the neonate was not tested immediately after birth. However, the mother's clinical condition deteriorated postpartum, she received intensive care and died on day 11 postpartum. Given the two prior COVID 19 positive reports of the mother (though the most recent report was negative), a strong suspicion of COVID 19 related aetiology as a cause of maternal death was kept. Therefore, the neonate was tested late, on day 14 of life. Neonate tested positive for COVID 19, though the viral load as detected by $\mathrm{Ct}$ was below the detection cut off. Subsequent testing of the neonate though was advised, but the family did not come for follow-up as they 
Table 3 Comparative between COVID 19 positive and COVID 19 negative neonates $(N=65)$

\begin{tabular}{|c|c|c|c|c|c|}
\hline S. No & Variable & $\begin{array}{l}\text { COVID } 19 \text { positive neonates } \\
N(\%) N=7\end{array}$ & $\begin{array}{l}\text { COVID } 19 \text { negative neonates } \\
N(\%) N=58\end{array}$ & $\mathrm{RR}(95 \% \mathrm{CI})$ & $p$ value \\
\hline 1. & Gestation & $36.3 \pm 2.04$ & $36.47 \pm 2.71$ & - & 0.94 \\
\hline 2. & Birth weight & $2452 \pm 870$ & $2542 \pm 726$ & - & 0.48 \\
\hline 3. & Female gender & $4(57.1 \%)$ & $32(57.1 \%)$ & 0.64 (0.19 to 2.1$)$ & 0.25 \\
\hline 4. & Mode of delivery (LSCS) & $3(44.4 \%)$ & $22(39.2 \%)$ & $0.65(0.18$ to 2.25$)$ & 0.25 \\
\hline 5. & Birth weight $\mathrm{z}$ scores (mean SD) & $-0.49 \pm 1.09$ & $-0.87 \pm 0.75$ & & 0.19 \\
\hline \multirow[t]{6}{*}{6.} & Gestation (weeks) & & & & \\
\hline & Term $(>37)$ & $4(66.6 \%)$ & $28(48.2 \%)$ & $2.33(0.63$ to 8.53$)$ & 0.10 \\
\hline & Late preterm $(34$ to $36+6)$ & 1 & 18 & & \\
\hline & Moderate preterm $(32$ to $33+5)$ & 1 & 10 & & \\
\hline & Very preterm $(28$ to $31+5)$ & 1 & 03 & & \\
\hline & Extreme preterm $(<28$ weeks $)$ & 0 & 1 & & \\
\hline \multirow[t]{5}{*}{7.} & Birth weight & & & & \\
\hline & $<1000 \mathrm{~g}$ & 0 & 1 & $1.56(0.46$ to 5.2$)$ & 0.24 \\
\hline & 1000 to $1500 \mathrm{~g}$ & 1 & 4 & & \\
\hline & 1500 to $2500 \mathrm{~g}$ & 3 & 25 & & \\
\hline & $>2500 \mathrm{~g}$ & $3(42.8 \%)$ & $18(32.1 \%)$ & & \\
\hline \multirow[t]{4}{*}{8.} & Delivery room resuscitation & & & & \\
\hline & Initial steps & $1(11.1 \%)$ & $8(14.2 \%)$ & $0.77(0.11$ to 5.49$)$ & 0.43 \\
\hline & PPV & 0 & & & \\
\hline & Intubation & 0 & & & \\
\hline \multirow[t]{4}{*}{9.} & Apgar score (at $1 \mathrm{~min}$ ) & & & & \\
\hline & $<4$ & 0 & $50(89.2 \%)$ & $0.96(0.14$ to 6.62$)$ & 0.45 \\
\hline & 4 to 6 & 1 & & & \\
\hline & $>6$ & $6(85.7 \%)$ & & & \\
\hline 10. & Separated at birth & $3(33.3 \%)$ & $10(17.2 \%)$ & $3.06(0.78$ to 11.81$)$ & 0.15 \\
\hline \multirow[t]{5}{*}{11.} & $\begin{array}{l}\text { Any respiratory support } \\
\text { Maximal respiratory support }\end{array}$ & $1(22.2 \%)$ & $16(28.5 \%)$ & $0.74(0.17$ to 3.25$)$ & 0.37 \\
\hline & Oxygen & 0 & 6 & & \\
\hline & CPAP & $1(14.2 \%)$ & 4 & & \\
\hline & Ventilation & 0 & 2 & & \\
\hline & Surfactant therapy & 0 & 1 & & \\
\hline 12. & Sepsis (clinical/culture positive) & 0 & $3(5.2 \%)$ & - & 0.48 \\
\hline \multirow[t]{10}{*}{13.} & Clinical signs & & & & \\
\hline & Asymptomatic & $6(85.7 \%)$ & $40(71.4 \%)$ & $1.34(0.30$ to 5.8$)$ & 0.37 \\
\hline & Symptomatic & $1(14.2 \%)$ & 16 & & \\
\hline & Respiratory distress & 1 & & & \\
\hline & Shock & 0 & & & \\
\hline & Feed intolerance & 0 & & & \\
\hline & Apnea & 0 & & & \\
\hline & Lethargy & 0 & & & \\
\hline & Comorbidities & & & & \\
\hline & Jaundice & 3 & 12 & & \\
\hline 14. & Mean duration of hospital stay (days) & $9.78 \pm 5.46$ & $7.73 \pm 4.92$ & - & 0.08 \\
\hline 15. & Number of deaths & 0 & $2(3.5 \%)$ & - & 0.57 \\
\hline 16. & Symptomatic mothers & $4(44.4 \%)$ & $38(67.8 \%)$ & $0.43(0.13$ to 1.4$)$ & 0.10 \\
\hline \multirow[t]{3}{*}{17.} & Maternal cycle thresholds (mean/SD) & & & & \\
\hline & E gene & $25.006 \pm 7.2$ & $27.16 \pm 7.46$ & - & 0.63 \\
\hline & RdRp gene & $19.69 \pm 3.87$ & $27.10 \pm 7.05$ & - & 0.08 \\
\hline 18. & Testing within $24 \mathrm{~h}$ & $2(22.2 \%)$ & $28(50.0 \%)$ & $0.33(0.0 .7$ to 1.4$)$ & 0.07 \\
\hline
\end{tabular}

*Does not include 1 abortion and 4 stillbirths

Data expressed as $N(\%)$ and mean $\pm \mathrm{SD}$ or median IQR

relocated to another city (after maternal death). Neonate 6 was born by caesarean delivery at 34 weeks gestation with a birth weight of $1800 \mathrm{~g}$. Neonate received NICU care due to prematurity and low birth weight. The mother also needed postpartum obstetric care and was kept under monitoring in the high dependency unit (HDU). On day 10 postpartum, the mother developed symptoms of fever and cough and was tested for
COVID 19, which was reported to be positive. Hence, the neonate was tested too on day 12 of life, which was reported positive. It is to be noted that mother and neonate were being cared for in the COVID block, where health care professionals followed the recommendation of donning complete PPE.

Possibility of horizontal transmission from asymptomatic NICU staff to neonate 2 and neonate 6 cannot be excluded 


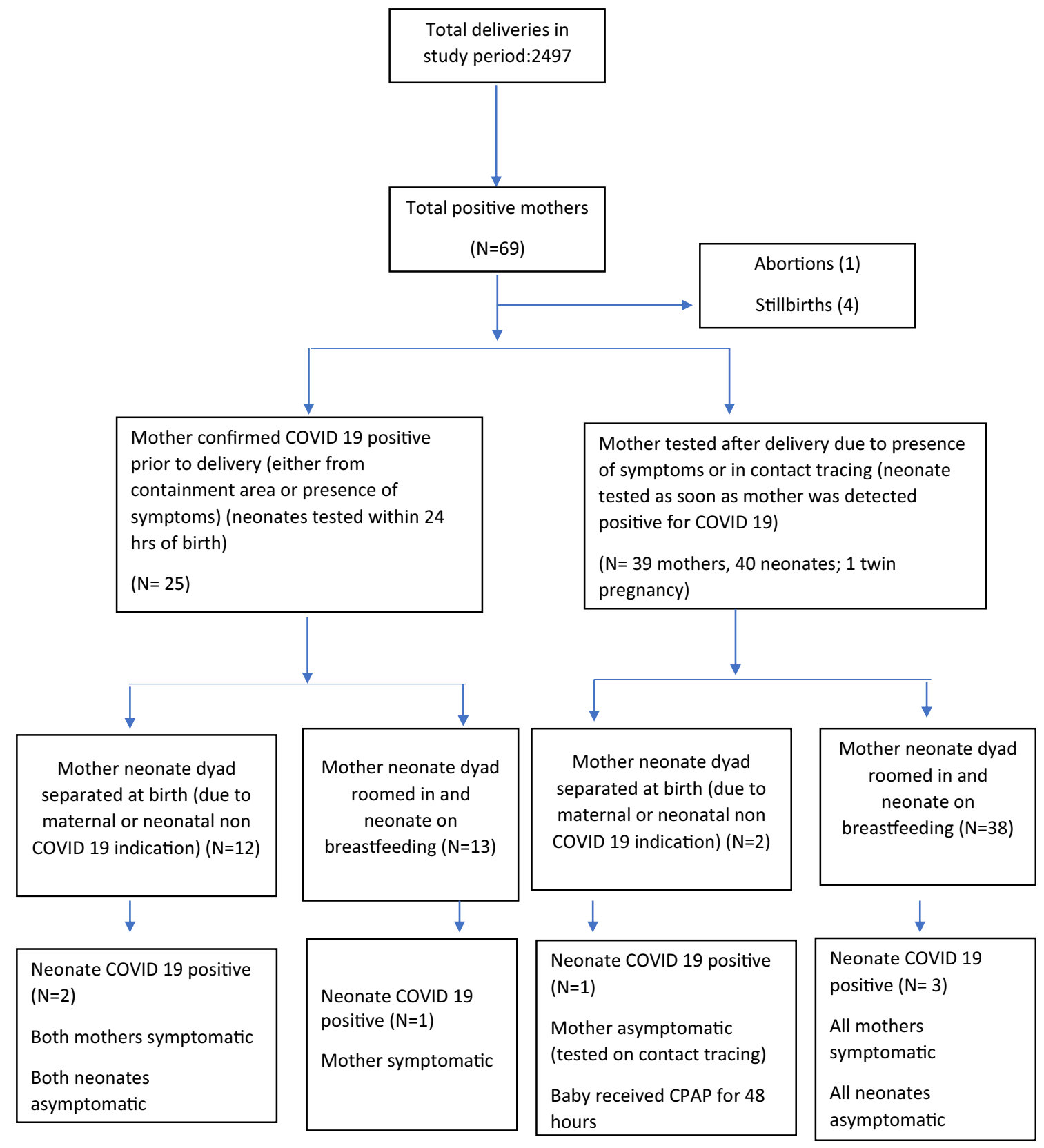

Fig. 2 Clinical profile of mothers and neonates positive for COVID 19

completely; however, it is to be noted that none of the other neonates admitted inside NICU and provided care by the same staff during that period, showed any specific symptoms or deterioration of the clinical condition, thus making it unlikely to be transmitted by a common caregiver. Moreover, in the unit, strict adherence to PPE and precautions during procedures was followed, to minimise the probability of transmission by health care providers. Universal surveillance of all health care providers entering NICU (including mothers) and admitted neonates in NICU continues to be an arguable point because of the uncertainty whether such surveillance would indeed prevent the illness in neonates and the psychological and economic burden which such a strategy may result in [43].

Shah et al [33] have described the categorization of maternal and neonatal COVID 19 illness as either confirmed, probable, possible, unlikely, or non-infected based on clinical symptoms, RTPCR positivity, type of samples tested for COVID 19 and timing of testing. As per this classification, in this cohort, all the mothers were classified as maternal infection during pregnancy as the virus was detected by PCR in respiratory samples in all of them. None of the stillbirths or abortions can be classified as congenital infection with intrauterine fetal death due to COVID 19, as an autopsy or 
placental or fetal tissue examination could not be performed. One neonate is classified as confirmed congenital infection in a live-born neonate (tested positive by RTPCR within $24 \mathrm{~h}$ of birth). Since none of the neonatal samples was taken at birth or within $12 \mathrm{~h}$, none of the neonatal infection can be classified as a neonatal infection acquired intrapartum. Six neonates are classified as a probable neonatal infection acquired postpartum and 56 neonates are classified as not infected.

La Scola et al [30] have reported a strong correlation between $\mathrm{Ct}$ value and sample infectivity in a cell culture model in 183 samples. They concluded that patients with $\mathrm{Ct}$ values equal or above 34 do not excrete infectious viral particles. In the current study, the cycle threshold value of $<35$ was interpreted as positive for SARS-CoV-2 RNA. The mean Ct values of 26.5 and 25.0 of $\mathrm{E}$ and $\mathrm{RdRp}$ genes respectively are consistent with some of the previous reports. However, notable is the case report by Vivanti et al where transplacental transmission is proven certain by placental and neonatal tests on multiple biosamples. The cycle load reported by Vivanti et al in a neonate on day 3 and 18 are lower (24 and 16 respectively) than those reported in the present study, thus indicating a higher viral load; neonate was symptomatic on day 3 with lethargy, irritability and opisthotonos [29].

The viral load of three neonates in the present study is comparable with the $\mathrm{Ct}$ reported from adults, thus raising the possibility that despite similar viral load as adults, manifestations are milder in neonates $[28,30,44,45] .$. Also notable is that viral load as indicated by $\mathrm{Ct}$ was highest in the first $72 \mathrm{~h}$ of life and almost below the detection cut off at the end of the second week, as observed in neonate 2, tested on day 14 of life. However, the results of the viral load need to be interpreted with caution as the number of neonates in whom viral load is available is small for us to deduce concrete conclusions. Cycle threshold levels were not being done in the initial phase of pandemic, due to which all COVID 19 positive neonates could not be assessed for the viral load.

The study from Italian municipality of Vo from Italy evaluated the viral genome equivalent inferred for cycle threshold data from RTPCR and indicated that asymptomatic and symptomatic participants did not differ in their viral load ( $p$ value 0.62 and 0.74 for $\mathrm{E}$ gene and RdRp gene) thus highlighting that health care workers should exercise enough prudence and practice the recommended PPE while handling the asymptomatic neonates too [46].

Opinions are conflicting regarding rooming-in and direct breastfeeding of a neonate born to COVID 19 positive mother, until she is tested negative $[10,22,47]$.

The World Health Organisation (WHO) recommendations [48] are based on the living systematic review by Centeno et al [49] which identified 12,198 records, of which 6945 were screened and 153 full-text reviews were included. The scientific brief by WHO recommends breastfeeding to be initiated or continued in suspected as well as confirmed COVID 19 infection. Current evidence on feeding practices and COVID 19 infection exists in the form of case reports, case series or family clusters $[10,50]$

One study reported viral particles of COVID 19 in breast milk; however, it was unclear through which route or source the neonate was infected [10]. RTPCR does not provide details regarding the viability or infectivity of the virus. No replication-competent virus was detectable in SARS CoV2 positive breast milk sample, in another report of 64 samples tested from 18 COVID 19 positive mothers [51]. Secretory IgA has been found in breast milk of COVID 19 infected mothers [52] but the strength and durability of the same have not been determined yet. To consider breast milk as potentially infectious, the presence of replicative COVID 19 virus in cell culture needs to be documented in future studies.

Therefore, the current data in the literature is insufficient to conclude the transmission in breastfeeding. In this existing scenario of a dearth of concrete evidence, benefits of breastfeeding vs harms of formula feeding by palade, cost and inability to maintain acceptable hygiene with top feed and hence higher incidence of morbidities and mortality due to top milk should be weighed upon, especially in developing nations. It is reassuring that despite most neonates being roomed-in with mother and breastfed since birth (51 of the 65), only 4 neonates (7.8\%) tested positive. The risk of transmission through mother and breastfeeding appears to be low if appropriate precautions are taken. This is all the more important for resource-limited settings where ensuring 3 to 6 feet distance between mother and neonate due to space constraints, availability of separate staff to provide expressed breast milk to the neonate and adequate supply of breast pumps and training of mother in manual expression may not be entirely possible.

Another consoling fact is that none of the neonates developed severe symptoms, 6 of the 7 (85\%) of COVID 19 positive neonates were asymptomatic and 1 neonate required CPAP for prematurity. The milder presentation in children and neonates has also been reported in the first systematic review in children by Liguora and colleagues, which included 62 studies and 3 reviews [53, 54].

The cause of distress was attributed to prematurity (respiratory distress syndrome). The radiological evaluation (chest $\mathrm{X}$-Ray) was normal for the neonate who needed CPAP as respiratory support. Comorbidities like meconium aspiration syndrome have been reported in previous reports too by Gregorio et al who described the use of lung ultrasound in the monitoring of respiratory morbidities in 3 neonates with COVID 19 infection [55].

There was no mortality noted amongst the 7 COVID 19 positive neonates and all remained haemodynamically stable throughout the hospital stay. However, we are not certain that the two neonatal deaths were not due to COVID 19 infection because the relevant samples like placental and neonatal tissues could not be examined and a neonatal autopsy could not 
be performed. Low rates of neonatal morbidity and mortality are comparable with the existing evidence, as reported by a systematic review of 9 studies by Smith et al.

The case for universal screening of antenatal mothers expecting a delivery, as advocated by few authors [56] is strong since, in this cohort too, more than $50 \%$ mothers became symptomatic in the post-partum period (varying from day 3 to day 11). Early identification in the pre-symptomatic stage may help in better strategic planning of maternal and neonate care to prevent COVID 19 transmission.

$40 \%$ rate of preterm deliveries is higher than the preCOVID preterm rate of 15 to $18 \%$ from the institute. Prematurity as a possible consequence of COVID 19 infection remains to be resolved in further studies. Also being a referral centre for high-risk pregnancies, delivery by caesarean delivery rate of $40 \%$ is higher than reported earlier; however, COVID 19 status of the mother did not determine the mode of delivery in the unit, the most common cause of caesarean delivery being fetal distress. Increased rates of preterm births and caesarean deliveries have also been observed in previous reports on maternal and neonatal COVID 19 infection. Preterm rate of as high as $63 \%$ and caesarean delivery rate of $80 \%$ has been described in the systematic review of 9 studies by Smith et al. [38] Noteworthy is that the rate of stillbirths in this cohort (57 per 1000 total births) is higher than preCOVID 19 rate of 34 per 1000 total births. Though the causative association with COVID 19 is uncertain, this aspect of COVID 19 effect on pregnancy outcomes needs to be evaluated in further studies.

The international database such as EPICENTRE which will register the data of maternal and neonatal COVID 19 is an initiative which is likely to provide understanding into epidemiology, clinical presentation and outcomes of paediatric and neonatal SARS CoV 2 in this evolving pandemic of COVID 19 infection [57, 58].

The report has the strengths of elaborate clinical details and the fact that all neonates born to positive mothers could be tested (unlike previous reports where the proportion of neonates could not be tested). It is a single centre descriptive study with no selection bias. RTPCR, which is the current gold standard of testing was the method used to confirm COVID 19 infection. Information on the viral load by cycle threshold of specific SARS-CoV-2 infection makes it more robust. It provides critical insights into clinical and epidemiological details in the perinatal period, the viral load and infectivity of the cases and hence adds to the sparse data presently available from developing countries. The data in context to roomingin and breastfeeding of neonates born to COVID 19 positive mothers provides important information that will have implications on clinical management of mother neonate dyad with COVID 19 in developing countries.

We acknowledge that the major limitation of this study is the lack of formal and serial evaluation of all the biosamples (amniotic fluid, placenta, newborn blood and cord blood both for RTPCR and serology) as needed for accurate diagnosis and international classification of COVID 19 infection in neonates. Though we have attempted to classify the 7 COVID positive neonates as per the classification criteria by Shah et al, interpretation is bounded by lack of the abovementioned biosamples. Placenta and fetal samples, amniotic fluid, umbilical cord blood and breast milk could not be tested for the disease in the current cohort; thus, the vertical transmission also remains underestimated in this cohort. Similarly, the effect of COVID 19 infection on stillbirths and prematurity rate as well as accurate diagnosis of the two neonatal deaths remains uncertain.

Also, due to the cycle load being available in fewer neonates, the association with maternal viral load and severity of neonatal symptoms remains confuted.

The study supports milder manifestation in COVID 19 infected neonates, despite similar viral load as adults. Risk of transmission of COVID 19 infection from mother to neonate by rooming-in and breastfeeding is low. In this cohort of a limited number of neonates, the maternal viral load does not appear to be associated with the positivity status or severity of the illness of neonate.

Authors' contributions PA and AY designed the study, collected the data, did the initial analyses and drafted the initial manuscript. PD helped in designing the study, supervised the conduct of the study, did the final analysis and reviewed and finalised the manuscript. SB and NG contributed to the collection of data, initial data analysis and reviewed and revised the initial manuscript. RG provided critical insights into study design, supervised the conduct of the study and critically reviewed the document.

\section{Compliance with ethical standards}

Conflict of interest The authors declare that they have no conflict of interest.

Ethical approval The study was approved by the Institutional Ethics Committee vide letter number IEC/VMMC/SJH/Project/2020-07/CC-21.

\section{References}

1. World Health Organization (WHO). WHO coronavirus disease 2019 (COVID-19) situation report 46. Available at: https://www. who.int/docs/default source/coronaviruse/situationreports/ 20200306-sitrep-46-covid-19.pdf? sfvrsn1/496b04adf_2. Accessed 24th May 2020

2. Zeng L, Xia S, Yuan W et al (2020) Neonatal early-onset infection with SARS-CoV-2 in 33 neonates born to mothers with COVID-19 in Wuhan, China. JAMA Pediatr. https://doi.org/10.1001/ jamapediatrics.2020.0878

3. Chen H, Guo J, Wang C, Luo F, Yu X, Zhang W, Li J, Zhao D, Xu D, Gong Q, Liao J, Yang H, Hou W, Zhang Y (2020) Clinical characteristics and intrauterine vertical transmission potential of COVID-19 infection in nine pregnant women: a retrospective review of medical records. Lancet. 395:809-815 
4. Zhang L, Jiang Y, Wei M et al (2020) Analysis of the pregnancy outcomes in pregnant women with COVID-19 in Hubei Province. Zhonghua Fu Chan Ke Za Zhi 55:E009

5. Fan C, Lei D, Fang C et al (2020. In press) Perinatal transmission of COVID-19 associated SARS-CoV-2: Should we worry? Clin Infect Dis. https://doi.org/10.1093/cid/ciaa226

6. Chen S, Huang B, Luo D et al (2020) Pregnant women with new coronavirus infection: clinical characteristics and placental pathological analysis of three cases. Zhonghua Bing Li Xue Za Zhi 49: E005

7. Chen Y, Peng H, Wang L, Zhao Y, Zeng L, Gao H et al (2020) Infants born to mothers with a new coronavirus (COVID-19). Front Pediatr. In press

8. Zamaniyan M, Ebadi A, Aghajanpoor MS (2020) Preterm delivery in pregnant woman with critical COVID-19 pneumonia and vertical transmission. Prenat Diagn. https://doi.org/10.1002/pd.5713

9. Sisman J, Jaleel MA, Moreno W et al (2020) Intrauterine transmission of SARS-COV-2 infection in a preterm infant [published online ahead of print, 2020 Jul 10]. Pediatr Infect Dis J. https://doi.org/ 10.1097/INF.0000000000002815

10. Costa S, Posteraro B, Marchetti S et al (2020) Excretion of SARSCoV-2 in human breast milk [published online ahead of print, 2020 Jun 2]. Clin Microbiol Infect:S1198-743X(20)30304-9

11. Buonsenso D, Costa S, Sanguinetti M, Cattani P, Posteraro B, Marchetti S, Carducci B, Lanzone A, Tamburrini E, Vento G, Valentini P (2020) Neonatal late-onset infection with severe acute respiratory syndrome coronavirus 2 . Am J Perinatol 37(8):869-872

12. Watson J, Whiting PF, Brush JE (2020) Interpreting a COVID-19 test result. BMJ 369:m1808

13. Ellington S, Strid P, Tong VT et al (2020) Characteristics of Women of reproductive age with laboratory-confirmed SARSCoV-2 Infection by pregnancy status - United States, January 22June 7, 2020. MMWR Morb Mortal Wkly Rep 69(25):769-775

14. Badr DA, Mattern J, Carlin A, Cordier A-G, Maillart E, El Hachem L, El Kenz H, Andronikof M, De Bels D, Damoisel C, Preseau T, Vignes D, Cannie MM, Vauloup-Fellous C, Fils J-F, Benachi A, Jani JC, Vivanti AJ (2020) Are clinical outcomes worse for pregnant women $\geq 20$ weeks' gestation infected with COVID-19? A multicenter case-control study with propensity score matching. Am J Obstet Gynecol. https://doi.org/10.1016/j.ajog.2020.07.045

15. Abbas AR, Abbas A, Ali Y, Memon SF (2020) Important considerations regarding the future management of coronavirus (COVID19). Int J Surg 79:6-7

16. Liu F, Liu H, Li J, Hou L, Lan W, Wang D (2020) Clinicoradiological features and outcomes in pregnant women with COVID-19: compared with age-matched non-pregnant women. Electronic copy available at https://ssrn.com/abstract $=3556647$ (Preprint). (31 March 2020)

17. Blitz MJ, Grunebaum A, Tekbali A, Bornstein E, Rochelson B, Nimaroff $M$ et al (2020) Intensive Care Unit Admissions for Pregnant and Non-Pregnant Women with COVID-19. Am J Obstet Gynecol

18. Qiancheng X, Jian S, Lingling P, Lei H, Xiaogan J, Weihua L, Gang Y, Shirong L, Zhen W, GuoPing X, Lei Z, sixth batch of Anhui medical team aiding Wuhan for COVID-19 (2020) Coronavirus disease 2019 in pregnancy. Int J Infect Dis 95:376383

19. Vivanti AJ, Mattern J, Vauloup-Fellous C, Jani J, Rigonnot L, El Hachem L et al (2020) Retrospective description of pregnant women infected with severe acute respiratory syndrome coronavirus 2 , France. Emerg Infect Dis

20. Hantoushzadeh S, Shamshirsaz AA, Aleyasin A, Seferovic MD, Aski SK, Arian SE, Pooransari P, Ghotbizadeh F, Aalipour S, Soleimani Z, Naemi M, Molaei B, Ahangari R, Salehi M, Oskoei AD, Pirozan P, Darkhaneh RF, Laki MG, Farani AK, Atrak S, Miri MM, Kouchek M, Shojaei S, Hadavand F, Keikha F, Hosseini MS,
Borna S, Ariana S, Shariat M, Fatemi A, Nouri B, Nekooghadam SM, Aagaard K (2020) Maternal death due to COVID-19 disease. Am J Obstet Gynecol 223:109.e1-109.e16

21. Austin PC (2011) An introduction to propensity score methods for reducing the effects of confounding in observational studies. Multivar Behav Res 46(3):399-424

22. Dong Y, Chi X, Huang H et al (2020) Antibodies in the breast milk of a maternal woman with COVID-19 [published online ahead of print, 2020 Jun 18]. Emerg Microbes Infect:1-12

23. Dong L, Tian J, He SM et al (2020) Possible vertical transmission of SARS-COVID-2 from an infected mother to her newborn. JAMA. https://doi.org/10.1001/jama.2020.46211 In press

24. Zhu H, Wang L, Fang C, Peng S, Zhang L, Chang G, Xia S, Zhou W (2020) Clinical analysis of 10 neonates born to mothers with 2019-nCoV pneumonia. Transl Pediatr 9:51-60

25. Zhang Z-J, Yu X-J, Fu T et al (2020) Novel Coronavirus infection in newborn babies aged $<28$ days in China. Eur Respir J 55(6): 2000697

26. Zeng H, Xu C, Fan J et al (2020) Antibodies in Infants Born to Mothers With COVID-19 Pneumonia [published online ahead of print, 2020 Mar 26]. JAMA 323(18):1848-1849

27. Coronado Munoz A, Nawaratne U, McMann D (2020) Late-onset neonatal sepsis in a patient with COVID-19. N Engl J Med 382(19): e49

28. Murphy S. Newborn baby tests positive for coronavirus in London. Available at: https://www.theguardian.com/world/2020/mar/14/ newborn-baby-tests-positive-for-coronavirusin-london. Accessed March 15, 2020

29. Vivanti AJ, Vauloup-Fellous C, Prevot S et al (2020) Transplacental transmission of SARS-CoV-2 infection. Nat Commun 11(1):3572

30. La Scola B, Le Bideau M, Andreani J et al (2020) Viral RNA load as determined by cell culture as a management tool for discharge of SARS-CoV-2 patients from infectious disease wards. Eur J Clin Microbiol Infect Dis 39:1059-1061

31. Chawla D, Chirla D, Dalwai S et al (2020) Perinatal-neonatal management of COVID-19 infection-guidelines of the Federation of Obstetric and Gynaecological Societies of India (FOGSI), National Neonatology Forum of India (NNF), and Indian Academy of Pediatrics (IAP). Indian Pediatr 57(6):536-548

32. Wang L, Shi Y, Xiao T et al (2020) Chinese expert consensus on the perinatal and neonatal management for the prevention and control of the 2019 novel coronavirus infection (First edition). Ann Transl Med 8(3):47

33. Shah PS, Diambomba Y, Acharya G, Morris SK, Bitnun A (2020) Classification system and case definition for SARS-CoV-2 infection in pregnant women, fetuses, and neonates. Acta Obstet Gynecol Scand 99(5):565-568

34. Mimouni F, Lakshminrusimha S, Pearlman S, Raju T, Gallagher P, Mendlovic J (2020) Perinatal aspects on the Covid-19 pandemic: a practical resource for perinatal-neonatal specialists. J Perinatol 40(5):820-826

35. Instructions for LabGun COVID 19 RTPCR kit available at https:// www.fda.gov/media/137483/download (last accessed 23rd August 2020)

36. Alagarasu K, Choudhary ML, Lole KS, NIC Team Evaluation of $R d R p \& O R F-1 b$-nsp14-based real-time RT-PCR assays for confirmation of SARS-CoV-2 infection: An observational study [published online ahead of print, 2020 May 30]. Indian J Med Res: 2020. https://doi.org/10.4103/ijmr.IJMR_1256_20

37. Oncel MY, Akın IM, Kanburoglu MK et al (2020) A multicenter study on epidemiological and clinical characteristics of 125 newborns born to women infected with COVID-19 by Turkish Neonatal Society [published online ahead of print, 2020 Aug 10] [published correction appears in Eur J Pediatr. 2020 Aug 22;]. Eur J Pediatr:1-10. https://doi.org/10.1007/s00431-020-03767-5 
38. Smith V, Seo D, Warty R et al (2020) Maternal and neonatal outcomes associated with COVID-19 infection: a systematic review. PLoS One 15(6):e0234187. https://doi.org/10.1371/journal.pone. 0234187

39. Martínez-Perez O, Vouga M, Cruz Melguizo S, Forcen Acebal L, Panchaud A, Muñoz-Chápuli M, Baud D (2020) Association between mode of delivery among pregnant women with COVID-19 and maternal and neonatal outcomes in Spain. JAMA. 324:296. https://doi.org/10.1001/jama.2020.10125

40. Kimberlin DW, Stagno S (2020) Can SARS-CoV-2 infection be acquired in utero?: more definitive evidence is needed. JAMA. Epub 2020 Mar 26. doi: 10.1001/jama.2020.4868. 6. Dong L, Tian J, He S, et al. Possible vertical transmission of SARS-CoV-2 from an infected mother to her newborn. JAMA. https://doi.org/10. 1001/jama.2020.4621

41. Li Y, Zhao R, Zheng S, Chen X, Wang J, Sheng X, Zhou J, Cai H, Fang Q, Yu F, Fan J, Xu K, Chen Y, Sheng J (2020) Lack of vertical transmission of severe acute respiratory syndrome coronavirus 2, China. Emerg Infect Dis 26:1335-1336

42. Schwartz DA (2020) An analysis of 38 pregnant women with COVID-19, their newborn infants, and maternal-fetal transmission of SARS-CoV-2: maternal coronavirus infections and pregnancy outcomes. Arch Pathol Lab Med. https://doi.org/10.5858/arpa. 2020-0901-SA

43. De Winter JP, De Luca D, Tingay DG (2020) COVID-19 surveillance for all newborns at the NICU; conditio sine qua non? [published online ahead of print, 2020 Aug 12]. Eur J Pediatr. https:// doi.org/10.1007/s00431-020-03773-7

44. Xu T, Chen C, Zhu Z et al (2020) Clinical features and dynamics of viral load in imported and non-imported patients with COVID-19. Int J Infect Dis 94:68-71

45. Zhang W, Du RH, Li B et al (2020) Molecular and serological investigation of 2019-nCoV infected patients: implication of multiple shedding routes. Emerg Microbes Infect 9(1):386-389. https:// doi.org/10.1080/22221751.2020.1729071

46. Lavezzo E, Franchin E, Ciavarella C et al (2020) Suppression of a SARS-CoV-2 outbreak in the Italian municipality of $\mathrm{Vo}^{\prime}$ ' [published online ahead of print, 2020 Jun 30]. Nature. https://doi.org/ 10.1038/s41586-020-2488-1

47. Stuebe A (2020) Should infants be separated from mothers with COVID-19? First, do no harm. Breastfeed Med 15(5):351-352

48. Breastfeeding and COVID 19 scientific brief, WHO/2019 nCoV/Sci_Brief/Breastfeeding/2020.1. https://www.who.int/ publications/i/item/10665332639. Accessed on 18th August 2020
49. Centeno-Tablante E, Medina-Rivera M, Finkelstein JL, RaycoSolon P, Garcia-Casal MN, Ghezzi-Kopel K, Rogers L, PeñaRosas JP, Mehta S (2020) Transmission of novel coronavirus-19 through breast milk and breastfeeding. A living systematic review of the evidence. PROSPERO. CRD42020178664.

50. Wu Z, McGoogan JM (2020) Characteristics of and important lessons from the coronavirus disease 2019 (COVID-19) outbreak in China: summary of a report of 72314 cases from the Chinese Center for Disease Control and Prevention. JAMA 24:1239. https://doi.org/10.1001/jama.2020.2648

51. Chambers C, Krogstad P, Bertrand K et al (2020) Evaluation for SARS-CoV-2 in breast milk from 18 infected women [published online ahead of print, 2020 Aug 19]. JAMA. https://doi.org/10. 1001/jama.2020.15580

52. Fox A, Marino J, Amanat F, Krammer F, Hahn-Holbrook J, ZollaPazner S, Powell RL. Evidence of a significant secretory-IgAdominant SARS-CoV-2 immune response in human milk following recovery from COVID-19. medRxiv preprint. https://doi.org/10. 1101/2020.05.04.20089995

53. Liguoro I, Pilotto C, Bonanni M, Ferrari ME, Pusiol A, Nocerino A, Vidal E, Cogo P (2020) SARS-COV-2 infection in children and newborns: a systematic review. Eur J Pediatr 179(7):1029-1046

54. Yoon SH, Kang JM, Ahn JG (2020) Clinical outcomes of 201 neonates born to mothers with COVID-19: a systematic review. Eur Rev Med Pharmacol Sci 24(14):7804-7815. https://doi.org/ 10.26355/eurrev_202007_22285

55. Gregorio-Hernández R, Escobar-Izquierdo AB, Cobas-Pazos J, Martínez-Gimeno A (2020) Point-of-care lung ultrasound in three neonates with COVID-19. Eur J Pediatr 179:1279-1285

56. Sutton D, Fuchs K, D'Alton M, Goffman D (2020) Universal screening for SARS-CoV-2 in women admitted for delivery. N Engl J Med 382(22):2163-2164

57. De Luca D, Rava L, Nadel S et al (2020) The EPICENTRE (ESPNIC Covid pEdiatric Neonatal Registry) initiative: background and protocol for the international SARS-CoV-2 infections registry. Eur J Pediatr 179(8):1271-1278

58. Ritz N, de Winter JP (2020) COVID-19 in children: patiently and critically evaluate the scientific evidence. Eur J Pediatr 179(8): $1179-1180$

Publisher's note Springer Nature remains neutral with regard to jurisdictional claims in published maps and institutional affiliations. 